\title{
Ogilvie's Syndrome With Caecal Perforation Following Caesarean Section: A Case Report
}

\author{
Ravichandran Nadarajaha, c, Julia Wan Chin Tan, Yin Ru Tan ${ }^{\mathrm{b}}$, Lay Kok Tan ${ }^{\mathrm{a}}$
}

\begin{abstract}
A 33-year-old healthy Caucasian woman underwent an emergency caesarean section at term for cephalopelvic disproportion. She developed abdominal distension and pain on first post operative day (POD) and was diagnosed clinically as having paralytic ileus. She refused an abdominal x-ray. Her condition improved with conservative management and she managed to pass flatus and have a bowel movement. She was discharged against medical advice on the sixth postoperative day. She presented to the emergency department on 14 th day post delivery with acute abdomen. Diagnosis of perforated viscus was made on abdominal x-ray. She underwent an emergency laparotomy. At laparotomy, a perforation in her caecum was found and a hemicolectomy and loop ilieostomy was performed. Unfortunately her postoperative recovery was complicated by wound dehiscence secondary to infection. She recovered and was discharged home 39 days after the emergency laparotomy. Ogilvie's syndrome can often be missed because the patient demonstrates signs of resolving ileus by passing flatus or having a bowel movement and have normal bowel sounds. Radiological confirmation of resolving ileus should be carried out if clinically the patient's abdomen is still distended. Timely recognition may prevent the complication of perforated caecum, which can occasionally prove fatal.
\end{abstract}

Keywords: Olgilvie syndrome; Caesaren section; Caecal perforation

\section{Introduction}

Ogilvie's syndrome or acute pseudo-obstruction of the large

\footnotetext{
Manuscript accepted for publication November 20, 2012

${ }^{a}$ Department of Obstetrics and Gynaecology Singapore General Hospital, Singapore

${ }^{b}$ National University of Singapore, Singapore

${ }^{\mathrm{C} C}$ Corresponding author: Ravichandran Nadarajah, Department of Obstetrics and Gynaecology Singapore General Hospital, Outram Road, 169608, Singapore. Email: ratadran@yahoo.com
}

doi: http://dx.doi.org/10.4021/jmc1042e bowel following caesarean section is a rare condition and the true incidence is unknown. Delay in diagnosis can lead to caecal perforation which is associated with significant morbidity and mortality.

\section{Case Report}

A 33-year-old, fit and healthy Caucasion woman, in her first pregnancy was admitted to the Obstetric Unit in spontaneous labor at term. Her pregnancy thus far was uneventful. She had a prolonged second stage of labor and after assessment was deemed to have cephalopelvic disproportion. She underwent an emergency caesarean section. The surgery was uneventful and her vital signs were stable intra and post operatively.

On the first postoperative day (POD), she complained of abdominal distension, pain and bloatedness. Her abdomen was tender and grossly distended but bowel sounds were audible although sluggish. Her temperature was normal and the laboratory results unremarkable. Paralytic ileus was diagnosed and conservative treatment with intravenous fluids and nasogastric tube was recommended. However, she declined the nasogastric tube insertion and an abdominal x-ray.

On second POD, the abdominal pain and distension had worsened and she consented to having a nasogastric tube inserted to facilitate decompression. However, she could not tolerate the nasogastric tube and it was removed on the same day. She was started on magnesium carbonate mixture and clear feeds. She continued to have persistent abdominal distention. Rectal tube insertion was attempted but failed. Again, she refused an abdominal x-ray. Baseline blood investigations including a full blood count and renal panel were within normal range. Her condition improved slightly and she insisted on being discharged on postoperative day 6 against medical advice.

The patient presented to the Emergency Department twelve days after delivery with drowsiness, lethargy and abdominal bloatedness. Clinically, she was noted to be lethargic and dehydrated, with altered mental state. Her abdomen was also noted to be extremely distended with tenderness 


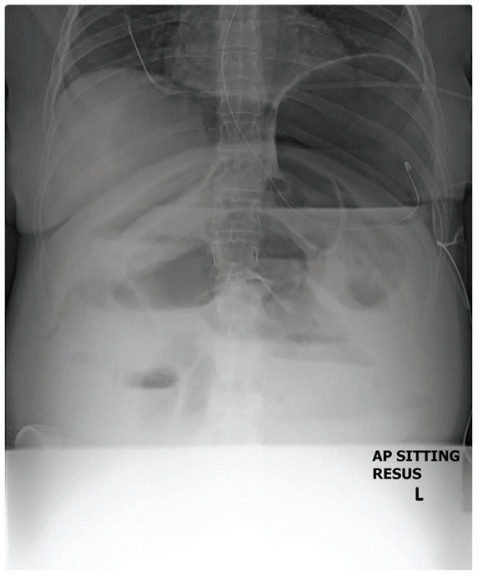

a

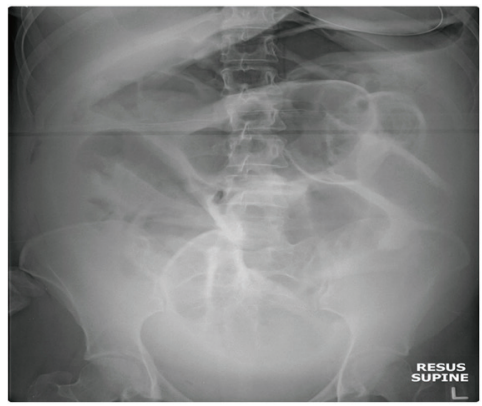

$\mathrm{C}$

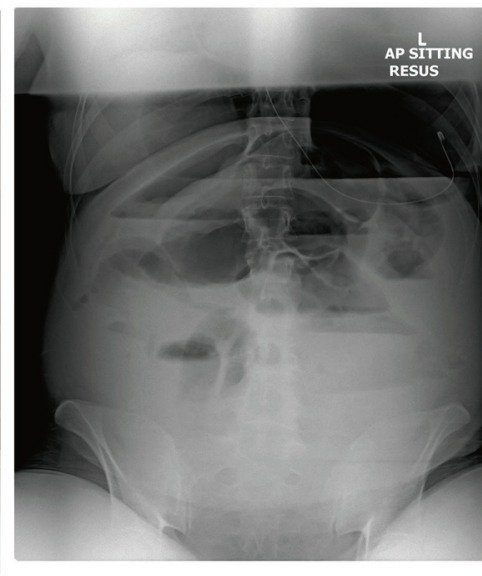

$\mathrm{b}$

Figure 1. Chest and abdomen. There is a large pneumoperitoneum as evident by continuous diaphragmatic outline, free intraperitoneal air lining the inferior margin of the liver and positive Rigler's sign. Generalised haziness in the lower abdomen with bulging flanks suggested presence of free fluid. Features are in keeping with recent viscus perforation. The small bowel loops are dilated and demonstrate wall thickening in left upper quadrant.

over the central abdomen.

Blood investigations revealed markedly elevated total white cell count of $29.9 \times 10^{9} / \mathrm{L}$, metabolic acidosis $(\mathrm{pH}$ : 7.21, BE: $-13.6 \mathrm{mmol} / \mathrm{L}$ ) and acute renal impairment (urea: $16.1 \mathrm{mmol} / \mathrm{L}$, creatinine $99 \mathrm{Umol} / \mathrm{L}$ ). Chest and abdominal $\mathrm{x}$-ray revealed a large pneumoperitoneum (Fig. 1a, b) and evidence of free fluid (Fig. 1c) in the peritoneal cavity with positive Rigler's sign suggestive of a perforated viscus. A decision was made for an emergency laparotomy.

Intra operatively, 4.8 litres of faeco-purulent fluid was encountered on entering the abdomen. There was a $5 \mathrm{~cm}$ perforation seen at the anti-mesenteric border of the caecum, extending into the ascending colon. Fibrinous exudates and adhesions were noted in the entire abdomen and between small bowel loops, which were grossly dilated. In view of the findings, adhesiolysis and a limited right hemi-colectomy was performed with a creation of a double barrel stoma. Histology of the resected bowel revealed localized gangrenous necrosis with perforation $(3.2 \times 2 \mathrm{~cm})$ with fibrinopurulent serositis.
Post operatively, she was started on total parental nutrition (TPN) and was extubated on POD 3. Unfortunately, she subsequently developed abdominal dehiscence of her laparotomy incision on the 9th postoperative day, which required a further exploratory laparotomy. Intra operatively, pus was noted at the midline and LSCS wound, in the pelvis and both paracolic gutters secondary to partial dehiscence of ileal end of the double barrel stoma. An abdominal cavity washout was performed with reinforcement of stoma and temporary abdominal closure.

A second look laparotomy was performed on POD 11. There was a dehiscence of the colonic end of the stoma. A washout was performed with reinforcement of the stoma, followed by a mass closure of the abdomen. Both the midline and Pfannenstiel wounds were left to heal by secondary intention. A CT scan of the abdomen and pelvis performed on POD 18 which showed subphrenic abscess of $16.8 \times 9.5 \mathrm{~cm}$. This was managed conservatively with percutaneous drainage. Follow up CT scan on POD 27 confirmed resolution of the abscess. 
She was eventually discharged on POD 39.

\section{Discussion}

Acute colonic pseudo-obstruction or Ogilvie syndrome (OS) is a rare condition characterized by rapid, progressive and massive dilatation of the caecum and proximal colon in the absence of mechanical dilatation. If not decompressed, the patient is at risk of caecal perforation and fecal peritonitis which are associated with a high mortality rate [1].

The true incidence of OS is unknown as many mild cases resolved spontaneously and no reliable national or international data exist on its frequency [2]. OS can occur in any age, but it is generally more common in the elderly patients and in those with various medical conditions, such as sepsis and trauma, or those who undergo surgical abdominal and/or pelvic procedures [1]. The use of drugs such as syntocinon and opioid in cases of OS has also been reported but any causal relationship is unclear. This condition is more common in men with a male to female ratio of 1.5:1 [3]. In obstetrics, caesarean section seems to be the most common operative procedure associated with this syndrome [4-9]. There are cases reported after vaginal delivery [7], forceps delivery [10], caesarean hysterectomy [11], and during pregnancy with preterm labor, pre-eclampsia and multiple pregnancies [12-14]. However, there is no data on predisposing factors or any association with respect to ethnic group, parity and indication for caesarean section.

There is a lack of consensus with respect to the aetiology and pathogenesis of the condition. OS is thought to be most likely due to an imbalance between the sympathetic and parasympathetic colonic innervation. OS in obstetric patients has been attributed to declining levels of serum oestrogen following childbirth and resulting decreased parasympathetic tone [15]. Animal studies and retrospective data suggest the cut off value of $12 \mathrm{~cm}$ in diameter is associated with an increased risk of caecal perforation [3-18].

Symptoms normally occur between 2 to 12 days post caesarean section. Progressive abdominal distension (90$100 \%$ of cases) and abdominal pain $(80 \%)$ are the commonest symptoms [2]. In OS, bowel sounds may vary from normal to hyperactive or absent and patients can still pass small amounts of faecal fluid and flatus. Localised tenderness over the right iliac fossa suggested caecal perforation [16]. A plain abdominal $x$-ray is the most useful diagnostic test shows dilatation of the caecum and large bowel which tail off at splenic flexure or rectosigmoid junction and pneumoperitoneum confirm bowel perforation.

Our patient presented on the first POD with abdominal distension, and was above to pass flatus and have a bowel movement. A higher index of suspicion and the inclusion of OG in the differential diagnosis may have persuaded the patient to accept having an abdominal x-ray earlier which could have demonstrated the radiological signs of caecal distension. Our patient also highlights the risk of premature discharge before complete resolution of her symptoms, and also the need for counseling with regard to serious and potentially life threatening consequences in the event of a full blown Ogilvie syndrome. Continued close inpatient monitoring would have detected the progression of the condition from worsening symptoms and allowed for timely intervention before perforation occurred.

In patients with a caecal dilatation of less than $10 \mathrm{~cm}$ in diameter, the treatment is conservative and consists of keeping the patient nil by mouth, nasogastric tube suction and insertion of rectal tube. Any medication that can affect the colonic motility such as narcotic analgesia and anti-cholinergic should be stopped. Patients should respond to conservative treatment within 24 to 48 hours and the response to the treatment is measured by serial abdominal $\mathrm{x}$-rays and improvements in patient symptoms. Colonic decompression with medication or endoscopic intervention is used if patient fails to respond to conservative treatment.

Pharmacological agents that increase the colonic motility such as enema with laxative, erythromycin, metaclorpramide and cisapride have been used. However the results are inconsistent [2]. Neostigmine is the only drug shown to be useful with a good response in the treatment for OS [17].

Colonoscopic decompression may be used if conservative or medical treatment fails. The success rate varies from $61-78 \%$ with a risk of recurrence of 22 to $41 \%$. Colonoscopy can be difficult to perform due to poor bowel preparation and it carries the risk of bowel perforation in up to $3 \%$ of patients [18]. Caecostomy is a surgical method to decompress the colon in cases with impending perforation. This procedure is usually carried out at laparotomy, although laparoscopic or CT guided procedures have had some success. In patient with colonic perforation, surgical procedures usually involve hemicolectomy with a temporary diversion.

\section{Conclusion}

Ogilvie's syndrome is a rare post-operative complication that carries significant morbidity and mortality. It is often missed in obstetric patients or misdiagnosed as paralytic ileus due to similarities in presentation. This case highlights the importance of high index of suspicion for OS in patients with abdominal distension following surgical procedures despite falsely reassuring presence of bowel sounds and passage of flatus, and the significant morbidity that could arise from a fully evolved Ogilvie syndrome. Early diagnosis and intervention are crucial to prevent catastrophic outcomes. Therefore, any patient who complains of increased abdominal distension should have early plain radiographs of the abdomen. If the colonic diameter is approaching $12 \mathrm{~cm}$, decompressive colonoscopy or nasogastric tube should be considered, and a multi-disciplinary approach involving the surgeons is ex- 
tremely important.

\section{Abbreviations}

POD: days after emergency laparotomy

\section{References}

1. Nakhgevany KB. Colonoscopic decompression of the colon in patients with Ogilvie's syndrome. Am J Surg. 1984;148(3):317-320.

2. CarpenterS, Holmstorm B. Ogilvie Syndrome. [www. eMedicine.com/med/topic2699.htm]

3. Vanek VW, Al-Salti M. Acute pseudo-obstruction of the colon (Ogilvie's syndrome). An analysis of 400 cases. Dis Colon Rectum. 1986;29(3):203-210.

4. Weber P, Heckel S, Hummel M, Dellenbach P. Ogilvie's syndrome after cesarean section. Apropos of 3 cases. Review of the literature. J Gynecol Obstet Biol Reprod (Paris) 1993; 22:653-8. [Article in French]

5. Moore JG, Gladstone NS, Lucas GW, Ravry MJ, Ansari AH. Successful management of post-cesarean-section acute pseudoobstruction of the colon (Ogilvie's syndrome) with colonoscopic decompression. A case report. J Reprod Med. 1986;31(10):1001-1004.

6. Rodriguez-Ballesteros R, Torres-Bautista A, TorresValadez F, Ruiz-Moreno JA. Ogilvie's syndrome in the postcesarean section patient. Int J Gynaecol Obstet. 1989;28(2):185-187.

7. Nanni G, Garbini A, Luchetti P, Ronconi P, Castagneto M. Ogilvie's syndrome (acute colonic pseudo-obstruction): review of the literature (October 1948 to March 1980) and report of four additional cases. Dis Colon
Rectum. 1982;25(2):157-166.

8. Roberts CA. Ogilvie's syndrome after cesarean delivery. J Obstet Gynecol Neonatal Nurs. 2000;29(3):239-246.

9. De M, Mandal A, Cooper JC. A case of Ogilvie's syndrome after caesarean section. J Obstet Gynaecol. 2002;22(6):686-687.

10. Kakarla A, Posnett H, Jain A, Ash A. Acute pseudoobstruction of the colon (Ogilvie's syndrome) following instrumental vaginal delivery. Int J Clin Pract. 2006;60(10):1303-1305.

11. Hamed AD, Dare FO. Ogilvie's syndrome. Int J Gynaecol Obstet. 1992;37(1):47-50.

12. Pecha RE, Danilewitz MD. Acute pseudo-obstruction of the colon (Ogilvie's syndrome) resulting from combination tocolytic therapy. Am J Gastroenterol. 1996;91(6):1265-1266.

13. Rieger NA, Lyon WJ, Bryce RL, Birrell SN. A case of acute colonic pseudoobstruction in pregnancy. Aust N Z J Obstet Gynaecol. 1996;36(3):363-365.

14. Kalu E, Fakokunde A, Jesudason M, Whitlow B. Acute colonic pseudo-obstruction (Ogilvie's Syndrome) following caesarean section for triplets. J Obstet Gynaecol. 2005;25(3):299-300.

15. Strecker JR, Jaluvka V. [Spontaneous cecum perforation following cesarean section]. Geburtshilfe Frauenheilkd. 1988;48(7):489-493.

16. Munro A. Large bowel obstruction. In: Wills BW, Paterson-Brown S,editors. Hamilton Bailey's Emergency Surgery. 13th ed. London: Hodder Arnold; 2000. p. 436-9.

17. Ponec RJ, Saunders MD, Kimmey MB. Neostigmine for the treatment of acute colonic pseudo-obstruction. $\mathrm{N}$ Engl J Med. 1999;341(3):137-141.

18. Eisen GM, Baron TH, Dominitz JA, Faigel DO, Goldstein JL, Johanson JF, Mallery JS, et al. Acute colonic pseudoobstruction. Gastrointest Endosc. 2002;56(6):789-792. 